\begin{tabular}{|c|c|}
\hline Title & Diversity changes of microbial communities into hospital surface environments \\
\hline Author(s) & $\begin{array}{l}\text { Y ano, Rika; Shimoda, Tomoko; W atanabe, Reina; Kuroki, Y asutoshi; Okubo, Torahiko; Nakamura, Shinji; Matsuo, } \\
\text { Junji; Y oshimura, Sadako; Y amaguchi, Hiroyuki }\end{array}$ \\
\hline Citation & $\begin{array}{l}\text { Journal of Infection and Chemotherapy, 23(7), 439-445 } \\
\text { https://doi.org/10.1016/.jiac.2017.03.016 }\end{array}$ \\
\hline Issue Date & $2017-07$ \\
\hline Doc URL & http:/hdl .handle.net/2115/71154 \\
\hline Rights & $\begin{array}{l}\text { () 2017. This manuscript version is made available under the CC-BY-NC-ND } 4.0 \text { license } \\
\mathrm{http}: / / \text { reativecommons.org/icenses/by-nc-nd/4.0/ }\end{array}$ \\
\hline Rights(URL) & http://creativecommons.org/icenses/by-nc-nd/4.0/ \\
\hline Type & article (author version) \\
\hline File Information & J Infect Chemother 2017.pdf \\
\hline
\end{tabular}

Instructions for use 


\section{Diversity changes of microbial communities into hospital surface environments}

2

3

4 Rika Yano $^{\mathrm{a}}$, Tomoko Shimoda ${ }^{\mathrm{a}}$, Reina Watanabe ${ }^{\mathrm{a}}$, Yasutoshi Kuroki ${ }^{\mathrm{b}}$, Okubo Torahiko ${ }^{\mathrm{b}}$,

$5 \quad$ Shinji Nakamura ${ }^{\mathrm{c}}$, Junji Matsuo ${ }^{\mathrm{b}}$, Sadako Yoshimura ${ }^{\mathrm{a}}$, Hiroyuki Yamaguchi ${ }^{\mathrm{a}}$ *

6

7

$8{ }^{a}$ Department of Fundamental Nursing, Faculty of Health Sciences, Hokkaido University,

9 Sapporo, Japan

$10{ }^{\mathrm{b}}$ Department of Medical Laboratory Science, Faculty of Health Sciences, Hokkaido

11 University, Sapporo, Japan

$12{ }^{\mathrm{c}}$ Laboratory of Morphology and Image Analysis, Biomedical Research Center, Juntendo

13 University Graduate School of Medicine, Tokyo, Japan

14

$15 *$ Corresponding author, Department of Medical Laboratory Science, Faculty of Health

16 Sciences, Hokkaido University, N12-W5, Kitaku, Sapporo 060-0812, Japan. 
17 Tel/Fax: +81-11-706-3326

18 E-mail address: hiroyuki@med.hokudai.ac.jp

19

20 E-mail:

21 Rika Yano: r-yano@med.hokudai.ac.jp

22 Tomoko Shimoda: tshimoda@hs.hokudai.ac.jp

23 Reina Watanabe: watanabe.reina@takenaka.co.jp

24 Yasutoshi Kuroki: y_k_5_9@hotmail.co.jp

25 Torahiko Okubo: t.okubo@hs.hokudai.ac.jp

26 Sadako Yoshimura: sadako@med.hokudai.ac.jp

27 Shinji Nakamura: shinji-n@juntendo.ac.jp

28 Junji Matsuo: matsuo@hs.hokudai.ac.jp

29 Hiroyuki Yamaguchi: hiroyuki@med.hokudai.ac.jp

30

31 Running title: Diversity changes of hospital environmental microbiome 


\section{Abstract}

35 Previous works have demonstrated considerable variability in hospital cleanliness in

36 Japan, suggesting that contamination is driven by factors that are currently poorly

37 controlled. We undertook 16S rRNA sequence analysis to study population structures of

38 hospital environmental microbiomes to see which factor(s) impacted contamination.

39 One hundred forty-four samples were collected from surfaces of three hospitals with

40 distinct sizes ("A": $>500$ beds, "B": $100-500$ beds, "C": $<100$ beds). Sample locations

41 of two ward types (Surgical and Internal) included patient room (multiple) (4BT),

42 patient overbed table (multiple) (4OT), patient room sink (multiple) (4S), patient room

43 bed table (single) (SBT), patient overbed table (single) (SOT), patient room sink

44 (single) (SS), nurse desk (ND), and nurse wagon (NW). Total DNA was extracted from

45 each sample, and the 50 samples that yielded sufficient DNA were used for further $16 \mathrm{~S}$

46 rRNA sequencing of hospital microbiome populations with cluster analysis. The number

47 of assigned bacterial OTU populations was significantly decreased in hospital " $\mathrm{C}$ " compared to the other hospitals. Cluster analysis of sampling locations revealed that the 
50 other hospitals was very similar and unusually skewed with a family,

51 Enterobacteriaceae. Interestingly, locations included patient area (4OT, 4BT, SBT) and

52 nurse area (ND), with a device (NW) bridging the two and a place (4S and SS) shared

53 between patients or visitors. We demonstrated diversity changes of hospital

54 environmental microbiomes with a skewed population, presumably by medical staff

55 pushing NWs or sinks shared by patients or visitors.

56

57

58 Keywords: Hygiene, Nosocomial infection, Hospital cleanliness, 16S rRNA sequence

59 analysis, Microbiome

60

61

62 


\section{Introduction}

64 CDC guidelines on controlling hospital-acquired infections (HAIs) prioritize

65 environmental cleanliness, while recommending precautions for contact between

66 patients and medical staff. In particular, the guidelines promote cleanliness of

67 high-contact surfaces such as machines placed around patients [1]. Bacterial

68 contamination of environmental surfaces is the leading cause of HAIs. Although

69 extensive measures to promote cleanliness are routinely undertaken, HAIs are a

70 persistent problem [2-8]. Thus, many hospitals implement monitoring of the

71 effectiveness of cleaning procedures, with visual assessment of surfaces, assessment of

72 residual fluorescent dye after cleaning, determination of aerobic colony-forming units,

73 or detection of ATP on surfaces [9].

74 Previous assessments of hospital cleanliness in Japan, using ATP bioluminescence

75 and stamp agar methods, revealed bacterial contamination to be highly variable and

76 independent of time or ward type [10]. No significant relationship was observed

77 between the number of patients or medical personnel in the hospital and organic or

78 microbiological contamination [10]. However, the physical properties of the surfaces 
79 that were sampled had a strong effect on contamination [11]. These findings suggest

80 that numerous currently uncontrolled factors contribute to HAIs in Japanese hospitals

81 [10-11]. Amplicon sequencing, targeting the hypervariable region of the bacterial

83 small-subunit ribosomal RNA gene (16S rRNA), is currently a widely used technique to

84 study environmental microbiomes. Data can be used quantitatively and qualitatively to

85 compare environments, as well as provide direct diagnostic information that can inform

86 therapeutic decisions $[12,13]$. Microbiome analysis also has been applied in a wide

87 range of research fields, including medicine, as well as ecology of indoor and marine

88 environments and urban public spaces such as subways [14-18]. Thus, microbiome

89 analysis is likely to provide pertinent data in attempts to understand the factors

90 controlling hospital cleanliness and HAIs.

91 In the present study, we undertook amplicon sequencing to visualize and compare

92 microbial population structures in three different hospitals, which differed in bed

93 numbers. 
96 Materials and methods

97 Hospitals

98 Because of their different sizes, three hospitals were selected, which have distinct

99 numbers of beds (hospital "A": >500 beds, hospital "B": 100-500, hospital "C": $<100$ ).

100 Both hospitals "A" and "B" are general hospitals with 30 and 21 clinical departments,

101 respectively, while hospital "C" specializes mainly in lung cancer treatment (including

102 antibiotic treatment to prevent infectious diseases after surgery) with only 5 clinical

103 departments. Because of distinct treatment policies, "Surgical" and "Internal" wards

104 were selected for sampling locations. For each of the wards, mean hospitalization

105 periods (days)/patient numbers are as follows: hospital "A": 24 (Surgical 33.2, Internal

106 14.8)/49.5 (Surgical 50.1, Internal 48), hospital "B": 15 (Surgical 16.3, Internal

107 13.7)/34.7 (Surgical 36.9, Internal 32.5), and hospital "C": 12.3 (Surgical 10.8, Internal

108 13.7)/21.1 (Surgical 18.5, Internal 23.4). These hospitals are located in the

109 Hokkaido-Tohoku area in Japan. Hospital features with relative ratios of patients with

110 lung cancer are summarized in Table 1 . The numbers of patients with lung cancer were 
111 obtained from data published as the hospital index, and the relative ratio of patients with

112 lung cancer among all patients was then estimated. Cleaning was similar in all hospitals

113 and followed CDC guidelines [1]. Cleaning was performed daily in several locations, as

114 shown in Fig. 1, according to the following protocol. In brief, floors were cleaned with

115 disinfectant (Fig. 1, asterisks). The overbed table or bed frame was wiped with detergent

116 and then treated with disinfectant (Fig. 1, arrowheads). The handrail or window frame

117 was also cleaned with detergent only (Fig. 1, arrows).

118

119 Sampling locations and swab collection

120 A total of 144 samples were collected from hospital environments. As mentioned above,

121 sampling locations belonging to two ward types [Surgical (Su) and Internal (In)]

122 included patient room (multiple) (4BT), patient overbed table (multiple) (4OT), patient

123 room sink (multiple) (4S), patient room bed table (single) (SBT), patient overbed table

124 (single) (SOT), patient room sink (single) (SS), nurse desk (ND), and nurse wagon

125 (NW). Samples were collected from these environmental surfaces on weekday mornings.

126 Sterile cotton swabs dipped in sterile saline were wiped over $400 \mathrm{~cm}^{2}$ at each location. 
127 To prevent contamination from hands, disposable sterilized plastic gloves were used for

128 all sample collections.

DNA extraction

131 Each cotton swab was intensely vortexed in sterile saline, and the resulting suspension

132 was then centrifuged, yielding pellets that were used for total genomic DNA extraction.

133 Extraction was performed using the QIAamp DNA kit (Qiagen, Valencia, CA, USA),

134 according to the manufacturer's instructions. DNA was eluted in $50 \mu 1$ of the elution

135 buffer supplied with the kit, and stored at $-20{ }^{\circ} \mathrm{C}$ until use. In addition, all laboratory

136 procedures were conducted in a safety cabinet with filtered airflow to prevent

137 cross-contamination.

138

139 Quality checking of extracted DNA

140 The quality of extracted DNA from each sample $(n=144)$ was confirmed by PCR

141 amplification using specific primers that target the 16S rRNA gene of Mycobacterium

142 sp., a species that inhabits most indoor and outdoor environments, including pond water 
143 and soil [18]. Band densities of electrophoresed PCR samples on $2 \%$ agarose gels were

144 quantified using ImageJ software. DNA samples from which Mycobacterium sp. 16S

145 rRNA could not be PCR amplified (n=94) were omitted from the following amplicon

146 sequence analysis because of insufficient DNA concentrations.

148 Amplicon sequence analysis

149 Analysis of samples that passed the quality check $(n=50)$ was performed according to

150 the following general protocols. First, DNA amplicons were amplified by specific

151 primer sets that target the V3-V4 region of SSU rRNA (supplied by Hokkaido System

152 Science Co., Ltd.); PCR products were resolved on $2 \%$ agarose gels and then purified.

153 After indexing PCR adaptor sequences had been added to the amplicons, a DNA library

154 for each sample was constructed, with a constant volume of amplicon solution (i.e.,

155 concentrations were not adjusted), to permit comparisons between samples of read

156 numbers produced by the Illumina 16S Metagenomic Sequencing Library Preparation

157 kit (Illumina). Libraries were sequenced on the MiSeq Illumina sequencer platform,

158 producing 300-bp paired-end reads. QIIME was used to process raw reads, with quality 
159 scores >30, and perform OTU (Operational Taxonomic Units) clustering. OTU

160 annotation was based on BLAST analysis with a baseline of $>90 \%$ similarity.

161 Metagenomic analysis including quality filtering, OTU production, taxonomic

162 classification, and phylogeny generation was all conducted by Hokkaido System

163 Science Co., Ltd. Cluster analysis was performed by using Cluster 3.0 for Mac OS X (C

164 Clustering Library 1.52). Phylogenetic trees generated from aligned population

165 structures were constructed and then visualized in Java TreeViewX (version 0.5.0).

166

167 Ethical considerations

168 Before samples were collected, the research design was explained to medical staff, and

169 informed consent was obtained orally from medical staff. Privacy and confidentiality of

170 personal information was protected according to the Helsinki Declaration [19]. Both the

171 Ethics Committee of Hokkaido University and the ethics review committee of each

172 hospital approved this study.

173

$174 \quad$ Statistical analysis 
175 Comparisons of assigned numbers into OTU units among hospitals were assessed by

176 single-factor ANOVA. A $p$ value of $<0.05$ was considered to be statistically significant.

177 All calculations were executed in Excel for Mac (2001) with Statcel3C.

178

179

180 Results

$181 \quad$ Validity of sampling and analysis

182 As described in the protocol outlined in Fig. 2, amplicon sequencing using Illumina

183 MiSeq was undertaken to compare microbiomes on surfaces in three hospitals ("A”,

184 "B", and "C") with distinct bed numbers. A total of 144 samples were randomly

185 collected from various hospital environmental surfaces, according to a general swabbing

186 protocol (see Methods). Based on the ability to PCR-amplify Mycobacterium 16S rRNA,

18750 samples were selected for next-generation sequencing targeting the hypervariable

188 (V3-V4) region of the bacterial 16S rRNA gene. Illumina sequencing yielded 8,948,588

189 sequences in total, with a mean of $178,971.76$ sequences per sample and a mean

190 sequence length of 419 bp. Sequence cleanup was performed using QIIME, creating 
191115,573 OTUs with a mean of 2,311 OTUs per sample. These data with sampling

192 information are deposited as supplementary data (Online Resource 1).

194 Hospital microbiome diversity and population structure

195 Microbial diversity was first compared among the three hospitals. Analyses of OTU

196 numbers revealed considerable diversity in each hospital, with 543 species in 23 phyla

197 in hospital "A", 519 species in 20 phyla in hospital "B", and 483 species in 17 phyla in

198 hospital "C". Hospital "C" was significantly less diverse than hospitals "A" and "B"

199 ( $p=0.0063)$ (Fig. 3). Population structure analysis among hospitals also showed that

200 species in family Enterobacteriaceae accounted for $78.49 \%$ of all OTUs identified only

201 in the samples of hospital "C" (data not shown). Thus, in contrast to the population

202 structure of samples from hospitals " $\mathrm{A}$ " and "B", hospital "C" appeared to show a

203 highly skewed population. Data were also analyzed by sampling location to understand

204 the factors responsible for the unique population structure with less diversity found in

205 samples from hospital "C". Cluster analysis among sampling locations revealed that the

206 population structure in almost all locations of hospital " $\mathrm{C}$ " and some locations in the 
207 other hospitals formed a cluster, being very similar and unusually skewed with a single

208 family, Enterobacteriaceae (Fig. 4, highlighted with an arrow). The locations included

209 patient area (4OT, 4BT, SBT) and nurse area (ND), with a device (NW) bridging the

210 locations and a place (4S and SS) shared between patients or visitors (Table 2).

\section{Discussion}

214 Since microbial contamination of the hospital environment likely contributes to the

215 spread of HAIs, surface disinfection and ward cleaning are typically prioritized to

216 maintain a clean clinical environment [19-23]. However, whether environmental

217 contamination has a role in the transmission of HAIs is poorly understood. Moreover,

218 there is little accurate epidemiological evidence based on metagenomic analysis to

219 support the belief that hospital cleanliness can specifically reduce the incidence of HAIs

$220 \quad[19-23]$.

221 Comparison among assigned numbers into OTU units of hospitals revealed

222 dramatic differences in diversity among hospitals, with hospital " $\mathrm{C}$ " being less diverse, 
224 (e.g., hospitalization periods, patient numbers, or cleaning protocol) than the use of 225 chemotherapy may be responsible, chemotherapy may lead to altered microbial 226 diversity. In fact, since cancer chemotherapy typically leads to physiological changes of 227 the human gut, with considerable alteration of the intestinal microbiome [24, 25], it 228 cannot be denied that microbial diversity may be the result of the influence of specific 229 drugs on patients' microbiomes, presumably with environmental spread of fecal bacteria 230 via toilets. Further studies, in particular gut microbiome analysis of patients, are needed 231 to clarify this association.

232 Furthermore, cluster analysis of sampling locations showed a cluster consisting of 233 samples from hospital "C" and samples from some locations of the other hospitals. The 234 results indicated that microbial population structures in these locations were very 235 similar and unusually skewed with a single family Enterobacteriaceae (unclassified 236 genera), members of which inhabit the human gut and can be associated with 237 antibiotic-resistant HAIs, spreading via the fecal-oral route [1-8, 21-25]. Interestingly, 238 the locations included patient area (4OT, 4BT, SBT) and nurse area (ND), with a device 
239 (NW) bridging the locations and a place (4S or SS) shared between patients or visitors.

240 Undoubtedly, washing hands with disinfectant is critical to reduce the prevalence of

241 nosocomial infections [26]. Hence, CDC guidelines promote not only cleanliness of

242 high-contact surfaces such as machines placed around patients, but also careful

243 handwashing with disinfectants for nurses after treating each patient $[1,26]$. However,

244 we found "sink" as well as "nurse wagon" in the cluster. Although the exact reason for

245 the unusually skewed cluster of Enterobacteriaceae remains to be clarified, it is likely

246 that unaware harmful behaviors of nurses while treating patient wastes or cleaning sinks

247 shared by patients or visitors result in the spread of bacteria originating from patients or

248 sinks to hospital wards. A recent study revealed that handwashing sinks were a possible

249 reservoir for KPC-2-producing Klebsiella oxytoca with a risk of hospital outbreak. Such

250 bacteria could persistently survive in the overflow trap of the sink and unknowingly be

251 transferred to individuals [26], even though cleaning according to CDC guidelines is

252 properly performed with disinfectant. Additionally, biofilms formed on the overflow

253 trap of the sink may facilitate bacterial survival [27]. Taken together, medical staff

254 pushing NW and sinks shared by patients or visitors likely not only changes the 
microbiota in hospital environments, but also spreads bacteria present in patient wastes

256 or the overflow trap of sinks to the hospital ward.

257 It may be unexpected that most of the OTUs identified from hospital "C" and

258 some samples from the other hospitals should belong to Gram-negative

259 Enterobacteriaceae, which have fragile cell walls. However, Escherichia coli can

260 survive on stainless steel surfaces without any nutrients for at least 100 days [28],

261 suggesting that Gram-negative bacteria can in fact remain viable for a long time on a

262 wide range of artificial surfaces. Although the exact reason why NWs became

263 contaminated with Gram-negative Enterobacteriaceae regardless of constant daily

264 cleaning remains unknown, there could be unrecognized factors responsible for

265 contamination on NW surface, which may be a significant factor determining the

266 relationships between hospital contamination and HAIs as well as contaminated sinks.

268 microbiomes with a skewed population, presumably by medical staff pushing NWs and

269 sinks shared between patients or visitors. This factor may help to explain variability in

270 the relationship between hospital contamination and HAIs. 


\section{Supporting Information}

274 Online Resource 1. Sample information (sampling location, ward type, hospital) and

275 basic data obtained from 16S rRNA amplicon sequencing. Sample numbers, $n=50$.

276

277

278 Declaration of interest

279 Consent for publication

$280 \quad$ Not applicable.

281

282

283 Availability of data and materials

284 The datasets generated during and/or analyzed during the current study are available

285 from the corresponding author upon reasonable request.

286 
289 The authors declare that they have no conflict interests.

290

291

292 Funding

293 This work was funded by the Japan Society for the Promotion of Science (JSPS),

294 Grant-in-Aid for Challenging Exploratory Research (No. 25670902 to RY).

295

296

297 Authors' contributions

298 The project was conceived by RY, TS, RW, SY, and HY. The samples were collected by

299 RY and TS. All laboratory work was conducted by RY, TS, YK, SN, TO, and JM.

300 Bioinformatics analysis was conducted by HY. The draft paper was critically edited by

301 RY, TS, TO, JM, and HY. RY and HY wrote the paper. 
303

\section{Acknowledgements}

305 We thank the staff members of the Departments of Medical Laboratory Science and 306 Nursing, Faculty of Health Sciences, Hokkaido University, for their assistance 307 throughout this study. 


\section{References}

310 [1] Siegel JD, Rhinehart E, Jackson M, Chiarello L. The Healthcare Infection Control

311 Practices Advisory Committee: Management of Multidrug-Resistant Organisms In

312 Healthcare Settings. Atlanta: Centers for Disease Control and Prevention. 2006.

313 [2] Samore MH, Venkataraman L, DeGirolami PC, Arbeit RD, Karchmer AW. Clinical

314 and molecular epidemiology of sporadic and clustered cases of nosocomial

$315 \quad$ Clostridium difficile diarrhea. Am J Med 1996; 100: 32-40.

316 [3] Hayden MK, Bonten MJ, Blom DW, Lyle EA, van de Vijver DA, Weinstein RA.

317 Reduction in acquisition of vancomycin-resistant Enterococcus after enforcement of

318 routine environmental cleaning measures. Clin Infect Dis 2006; 42: 1552-60.

319 [4] Hayden MK, Blom DW, Lyle EA, Moore CG, Weinstein RA. Risk of hand or glove 320 contamination after contact with patients colonized with vancomycin-resistant 321 Enterococcus or the colonized patient's environment. Infect Control Hosp Epidemiol $322 \quad 2008 ; 29: 149-54$.

323 [5] Martinez JA, Ruthazer R, Hansjosten K, Barefoot L, Snydman DR. Role of 
324 environmental contamination as a risk factor for acquisition of vancomycin-resistant

325 enterococci by in patients treated in a medical intensive care unit. Arch Intern Med

$326 \quad 2003 ; 163: 1905-12$.

327 [6] Huang SS, Datta R, Platt R. Risk of acquiring antibiotic-resistant bacteria from prior 328 room occupants. Arch Intern Med 2006; 166: 1945-51.

329 [7] Goodman ER, Platt R, Bass R, Onderdonk AB, Yokoe DS, Huang SS. Impact of an 330 environmental cleaning intervention on the presence of methicillin-resistant 331 Staphylococcus aureus and vancomycin-resistant enterococci on surfaces in intensive 332 care unit rooms. Infect Control Hosp Epidemiol 2008; 29: 593-9.

333 [8] Wu HM, Fornek M, Schwab KJ, Chapin AR, Gibson K, Schwab E. A norovirus 334 outbreak at a long term-care facility: the role of environmental surface 335 contamination. Infect Control Hosp Epidemiol 2005; 26: 802-10.

[9] Boyce JM. Environmental contamination makes an important contribution to 337 hospital infection. J Hosp Infect 2007; 65(Suppl. 2): 50-4.

338 [10] Watanabe R, Shimoda T, Yano R, Hayashi Y, Nakamura S, Matsuo J, et al. 339 Visualization of hospital cleanliness in three Japanese hospitals with a tendency 
$340 \quad$ toward long-term care. BMC Res Notes, 2014; 7: 121.

341 [11] Shimoda T, Yano R, Nakamura S, Yoshida M, Matsuo J, Yoshimura S, et al. ATP

342 bioluminescence values are significantly different depending upon material surface

343 properties of the sampling location in hospitals. BMC Res Notes 2015; 8: 807.

344 [12] Oulas A, Pavloudi C, Polymenakou P, Pavlopoulos GA, Papanikolaou N, Kotoulas

345 G, et al. Metagenomics: tools and insights for analyzing next-generation sequencing

346 data derived from biodiversity studies. Bioinform Biol Insights 2015; 9: 75-88.

347 [13] Vincent AT, Derome N, Boyle B, Culley AI, Charette SJ. Next-generation

348 sequencing (NGS) in the microbiological world: How to make the most of your

349 money. J Microbiol Methods 2016; pii: S0167-7012(16)30031-8.

350 [14] Jovel J, Patterson J, Wang W, Hotte N, O'Keefe S, Mitchel T, et al. Characterization

351 of the gut Microbiome using 16S or shotgun metagenomics. Front Microbiol 2016;

$352 \quad 7: 459$.

353 [15] Huang B, Fettweis JM, Brooks JP, Jefferson KK, Buck GA. The changing

$354 \quad$ landscape of the vaginal microbiome. Clin Lab Med 2014; 34:747-61.

355 [16] Prussin AJ, Marr LC, Bibby KJ. Challenges of studying viral aerosol 
metagenomics and communities in comparison with bacterial and fungal aerosols.

358 [17] Bik HM. Deciphering diversity and ecological function from marine metagenomes.

$359 \quad$ Biol Bull 2014, 227: 107-16.

360 [18] Afshinnekoo E, Meydan C, Chowdhury S, Jaroudi D, Boyer C, Bernstein N, et al.

361 Geospatial resolution of human and bacterial diversity with city-scale metagenomics.

$362 \quad$ Cell Syst 2015; 1: 72-7.

363 [19] Boyce JM, Potter-Bynoe G, Chenevert C, King T. Environmental contamination

364 due to methicillin-resistant Staphylococcus aureus: possible infection control

365 implications. Infect Control Hosp Epidemiol 1997; 18: 622-7.

366 [20] Dancer SJ. How do we assess hospital cleaning? A proposal for microbiological

367 standards for surface hygiene in hospitals. J Hosp Infect 2004; 56: 10-5.

368 [21] Dancer SJ. Importance of the environment in meticillin-resistant Staphylococcus

369 aureus acquisition: the case for hospital cleaning. Lancet Infect Dis 2008; 8: 101-13.

370 [22] Dancer SJ, White LF, Lamb J, Girvan EK, Robertson C. Measuring the effect of

371 enhanced cleaning in a UK hospital: a prospective cross-over study. BMC Med 2009; 
[23] N.P.S.A. The National Specifications for Cleanliness in the NHS: A Framework for

374 Setting and Measuring Performance Outcomes, London, The National Patient Safety $375 \quad$ Agency. 2007.

376 [24] Chase D, Goulder A, Zenhausern F, Monk B, Herbst-Kralovetz M. The vaginal and 377 gastrointestinal microbiomes in gynecologic cancers: a review of applications in 378 etiology, symptoms and treatment. Gynecol Oncol 2015; 1381: 190-200.

379 [25] Ciorba MA, Hallemeier CL, Stenson WF, Parikh PJ. Probiotics to prevent 380 gastrointestinal toxicity from cancer therapy: an interpretive review and call to action.

381 Curr Opin Support Palliat Care 2015; 9: 157-62.

382 [26] Won SP, Chou HC, Hsieh WS, Chen CY, Huang SM, Tsou KI, et al. Handwashing 383 program for the prevention of nosocomial infections in a neonatal intensive care unit. Infect Control Hosp Epidemiol 2004; 25:742-6. containing viable multiresistant organisms despite terminal cleaning on clinical surfaces in an intensive care unit. J Hosp Infect 2012; 80: 52-5. 
388 [28] Warnes SL, Highmore CJ, Keevil CW. Horizontal transfer of antibiotic resistance

389 genes on abiotic touch surfaces: implications for public health. MBio 2012; 3:

$390 \quad$ e00489-12.

391

392

393

394 


\section{Figure legends}

397 Fig. 1. Daily cleaning places in hospital aisles and patient rooms. Asterisks indicate 398 floors cleaned with disinfectant. Arrowheads indicate overbed tables or bed frames 399 wiped with detergent followed by treatment with disinfectant. Arrows indicate handrails 400 or window frames cleaned with detergent only

402 Fig. 2. Flowchart illustrating experimental protocols for sampling, quality checks and 403 sample selection, Illumina sequencing, and population analysis

405 Fig. 3. Differences in assigned numbers into OTU units (microbiome populations) 406 among hospitals. Asterisks indicate statistically significant differences $(p<0.05)$, 407 estimated by single-factor ANOVA 
409 Fig. 4. Cluster analysis of sampling locations shows population structures in almost all 410 locations of hospital " $\mathrm{C}$ " and some locations in the other hospitals clustered together 411 and were unusually skewed with Enterobacteriaceae 
Table 1 Hospital's size and summary

\begin{tabular}{|c|c|c|c|c|c|c|}
\hline $\begin{array}{c}\text { Hospital } \\
\text { (Speciality/Total bed number) }\end{array}$ & $\begin{array}{l}\text { Number of } \\
\text { clinical } \\
\text { department }\end{array}$ & Ward & $\begin{array}{l}\text { Bed number } \\
\text { per ward }\end{array}$ & $\begin{array}{c}\text { Average } \\
\text { hospitalization } \\
\text { periods (Days) }\end{array}$ & $\begin{array}{c}\text { Patient } \\
\text { numbers per } \\
\text { ward }\end{array}$ & $\begin{array}{l}\text { Relative ratio of } \\
\text { patients with } \\
\text { lung cancer }^{1}\end{array}$ \\
\hline $\begin{array}{c}\mathrm{A} \\
\text { (General hospital/500-1,000) }\end{array}$ & 30 & $\begin{array}{l}\text { Surgical } \\
\text { Internal }\end{array}$ & $\begin{array}{l}60 \\
60\end{array}$ & $\begin{array}{l}33.2 \\
14.8\end{array}$ & $\begin{array}{c}50.1 \\
48\end{array}$ & $\begin{array}{l}1 \\
1\end{array}$ \\
\hline $\begin{array}{c}\text { B } \\
\text { (General hospital/100-500) }\end{array}$ & 20 & $\begin{array}{l}\text { Surgical } \\
\text { Internal }\end{array}$ & $\begin{array}{l}46 \\
40\end{array}$ & $\begin{array}{l}16.3 \\
13.7\end{array}$ & $\begin{array}{l}36.9 \\
32.5\end{array}$ & $\begin{array}{l}0.3 \\
0.5\end{array}$ \\
\hline $\begin{array}{c}\text { C } \\
\text { (Lung cancer treatment/less than 100) }\end{array}$ & 5 & $\begin{array}{l}\text { Surgical } \\
\text { Internal }\end{array}$ & $\begin{array}{l}24 \\
25\end{array}$ & $\begin{array}{l}10.8 \\
13.7\end{array}$ & $\begin{array}{l}18.5 \\
23.4\end{array}$ & $\begin{array}{l}4.5 \\
2.4\end{array}$ \\
\hline
\end{tabular}

${ }^{1}$ ratio vs. Hospital A: patient numbers with lung cancer were obtained from data published as hospital index 
Figure 1

Hospital aisle

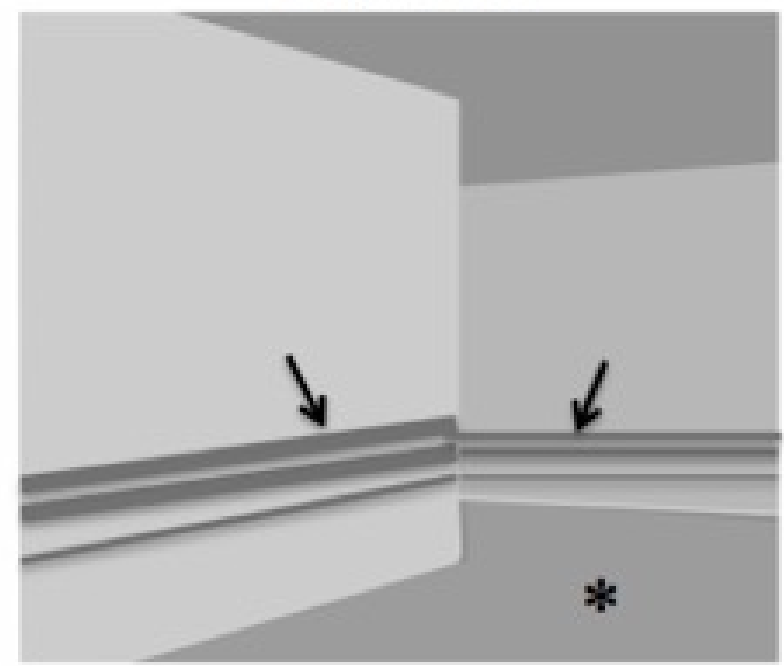

Hospital room

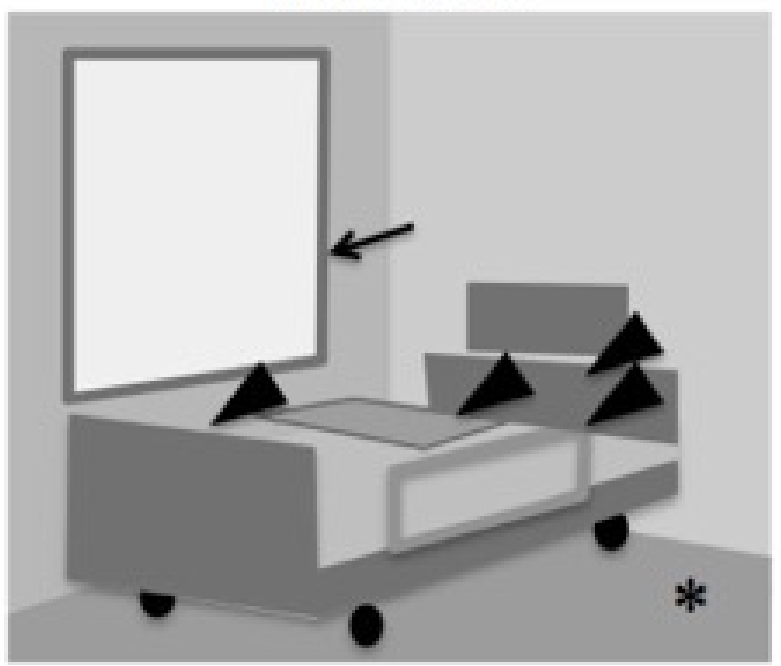


Figure 2

Quality check for sample DNAs and sample sellection

Collection of smear samples: $n=144$

$\downarrow$

Total DNA extraction

$\downarrow$

PCR amplification (target: Mycobacterium16SrDNA)

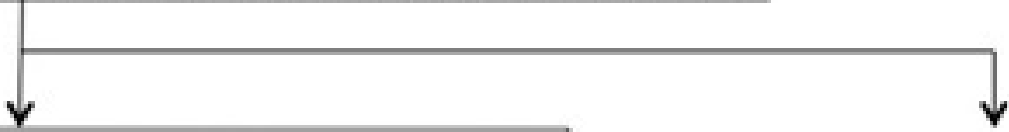

Yes, used for the following process: $n=50$

No, omitted: $\mathrm{n}=94$

Illumina Run

PCR amplification: $n=50$ (target: Bacterial 16SrDNA V3-V4 region)

$\downarrow$

Purification of amplified PCR products with addition of adapter sequences

$\downarrow$

MiSeq Illumina Run

$\downarrow$

Acquisition of read sequences

Population analysis

Clean-up of read sequences and confirmation( $>30$, Quality score), $n=50$

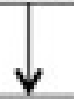

OTU (operational taxonomic unit) production

$\downarrow$

Annotation of OTU, $>90 \%$ similarity 
Figure 3

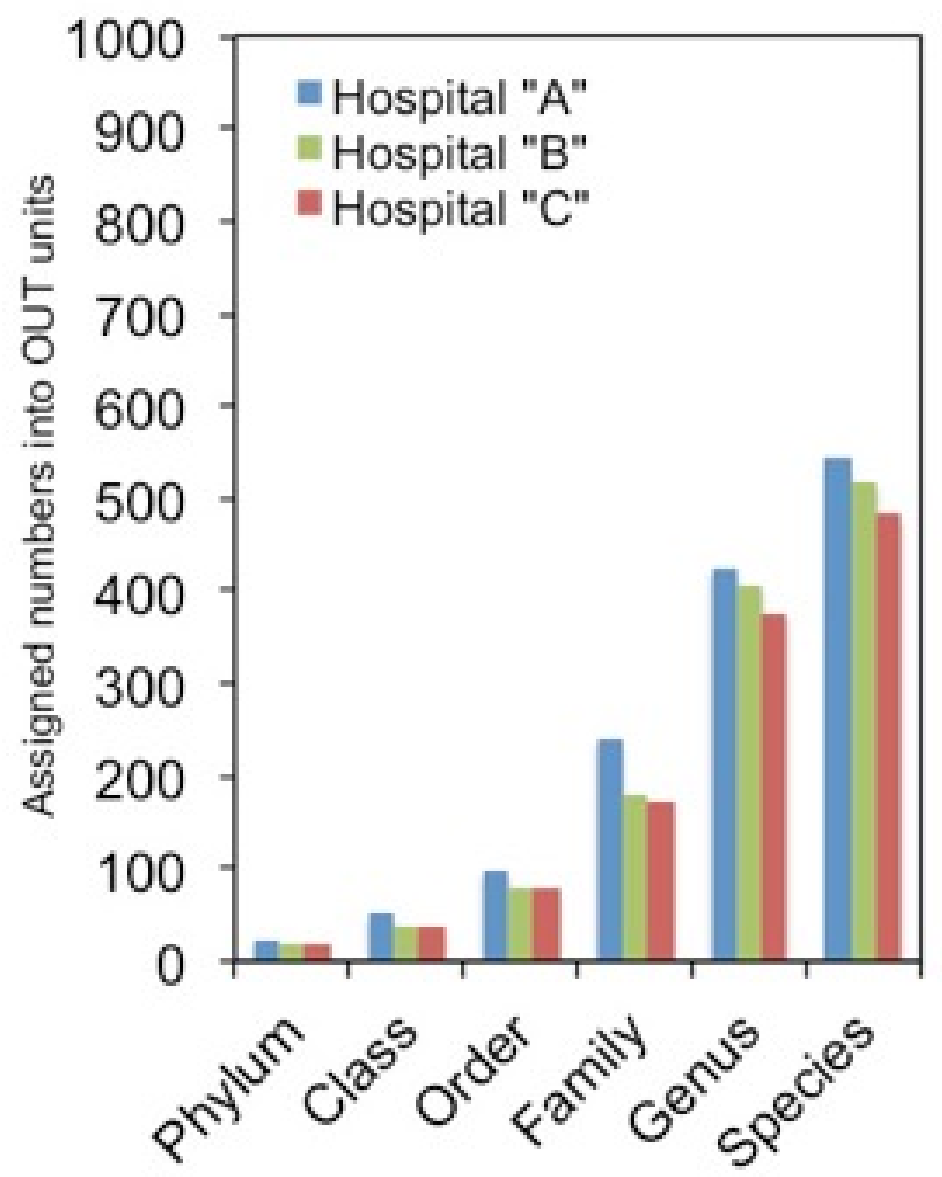

$p=0.0063^{*}$

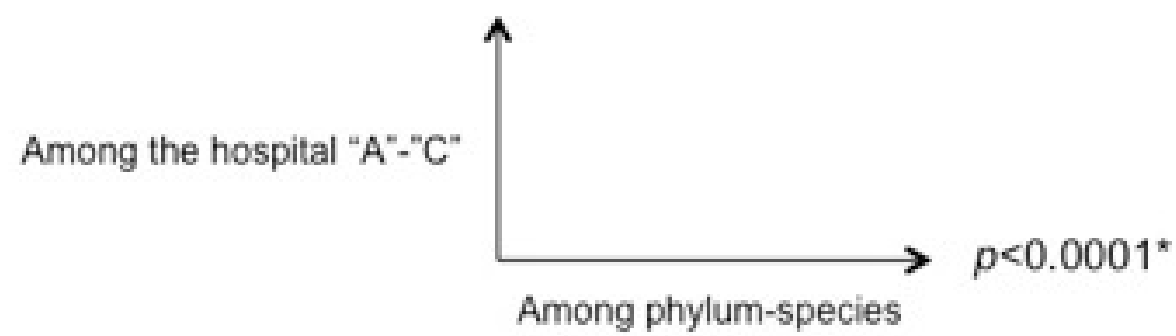


Figure 4

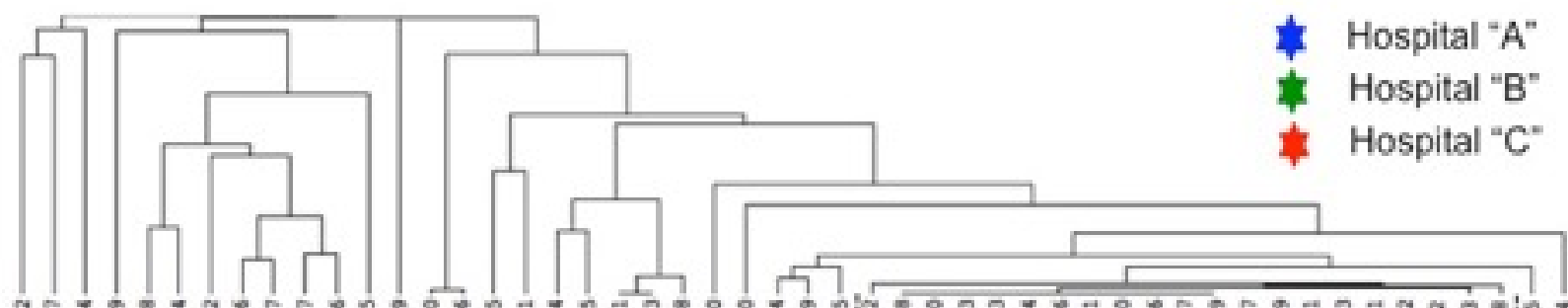

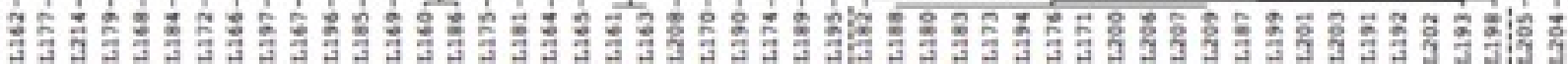

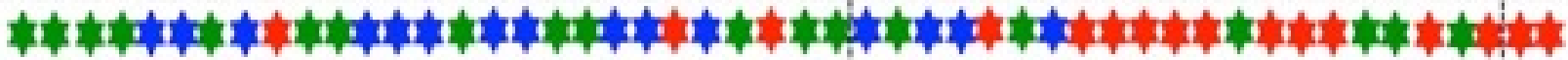

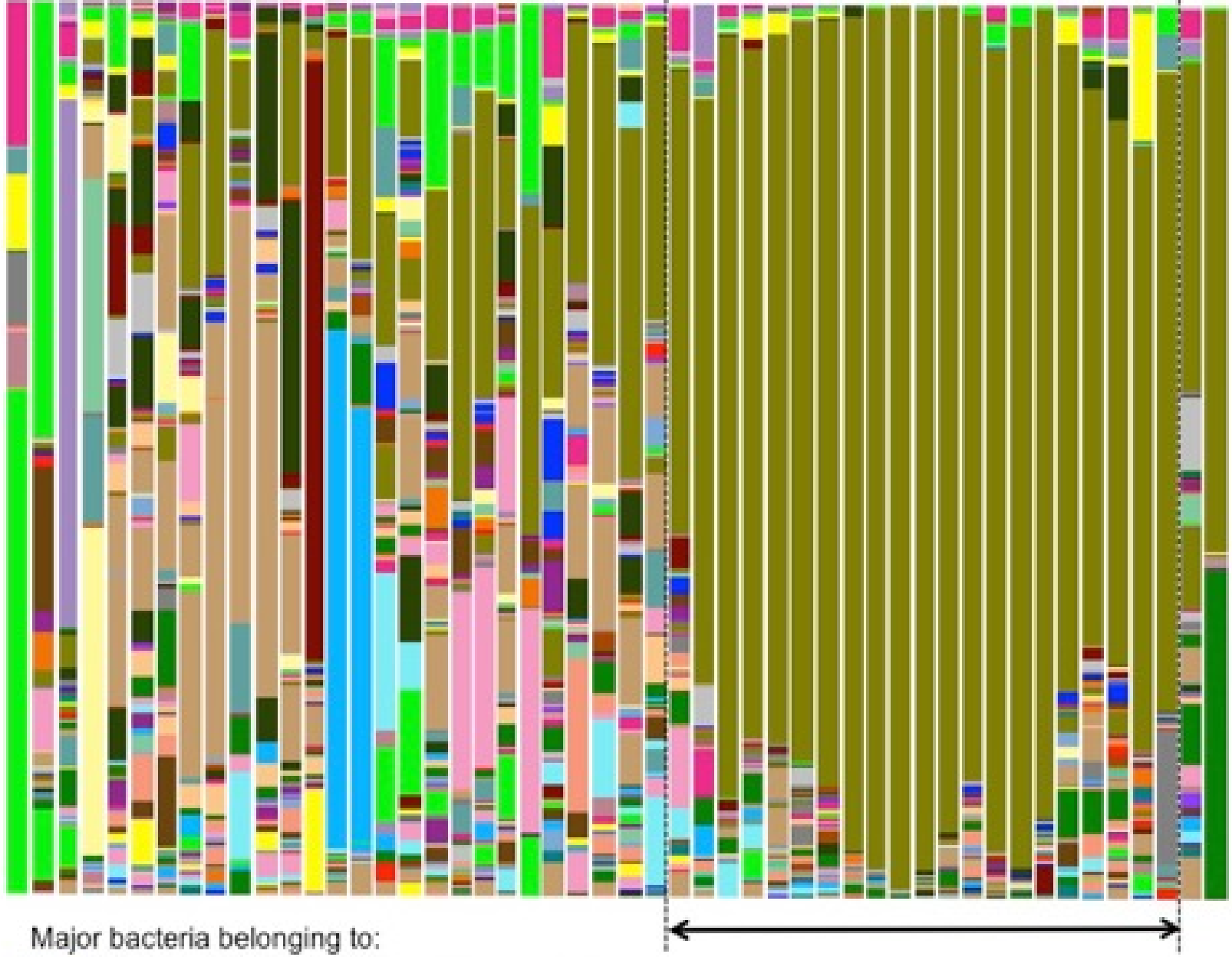

Unclassified genera into Enterobacteriaceae

Enhydrobacter sp.

Streptococcus sp.

Unclassified genera into Caulobacteraceae

Staphylococcus sp. 
Table 2 Hospital location and ward type of the samples being more similar to each other in a cluster

\begin{tabular}{cccc}
\hline Hospital & Sample number & Sampling location ${ }^{1}$ & Ward type $^{2}$ \\
\hline A & L176 & SBT & Su \\
A & L180 & $4 O T$ & Su \\
A & L182 & NW & Su \\
A & L183 & ND & Su \\
& & & \\
B & L187 & SBT & Su \\
B & L188 & $4 O T$ & Su \\
B & L191 & NW & In \\
B & L192 & 4BT & In \\
B & L193 & $4 S$ & In \\
B & L194 & $4 O T$ & In \\
C & L171 & NW & Su \\
C & L173 & SS & Su \\
C & L198 & 4BT & Su \\
C & L199 & SBT & Su \\
C & L200 & ND & Su \\
C & L201 & $4 O T$ & Su \\
C & L202 & SBT & Su \\
C & L203 & $4 S$ & Su \\
C & L206 & SS & In \\
C & L207 & $4 O T$ & In \\
C & L209 & $4 B T$ & In \\
\hline
\end{tabular}

${ }^{1}$ patient room (multiple), 4BT); patient overbed table (multiple), 4OT); patient room sink (multiple), 4S; patient room bed table (single), SBT; patient overbed table (single), SOT); patient room sink (single), SS); nurse desk, ND); nurse wagon (NW). ${ }^{2}$ Surgical, Su; Internal, In. 